\title{
Winkelsteife Implantate am Fersenbein, warum?
}

\author{
Hans Zwipp, Stefan Rammelt, Sven Barthel
}

\section{Zusammenfassung}

Die biomechanische Überlegenheit des winkelstabilen Calcaneusplättchens gegenüber dem SandersPlättchen ist noch nicht erwiesen. Die Reduktion einer additiven Spongiosaplastik von 53 auf 4\% mit einer damit verbundenen früheren Vollbelastung von 3 Wochen i.M. ist evident. Die eigene Anwendung des neuen Implantates in den letzten 2 Jahren war nur deshalb zurückhaltend, da bislang keine Titan-Molly15-Version zur Verfügung stand, die ein postoperatives CT problemlos ermöglicht.

\section{Einleitung}

Das Fersenbein ist nicht nur der größte aller tarsaler Knochen, sondern auch der am häufigsten gebrochene. Bei axialer Gewalteinwirkung kann der Calcaneus aufgrund seiner vorwiegend spongiösen Architektur erheblich kollabieren und massiv impaktiert werden. Er ist operationstechnisch schwierig aufzurichten, ebenso, wenn nicht gelenkübergreifend, $\mathrm{zu}$ retinieren und neigt wegen seiner schmächtigen Weichteilummantelung wie kaum ein anderer tarsaler Knochen zur Infektion. Diese Imponderabilien haben einen langen Weg zur Entwicklung eines optimierten Implantates bedingt.

\section{Implantatentwicklung}

Nach den Pionieren der operativen Frakturbehandlung wie Leriche (1929), Lenormant und Wilmoth (1929), Westhues

OP-JOURNAL 2004; 20: 32-36

(c) Georg Thieme Verlag KG Stuttgart · New York
(1935) und Merle d'Aubigné (1936) $[5,6,7,8]$, die mittels Spickdraht- und Schraubenosteosynthese oder Knochenspanunterfütterung Fersenbeinbrüche angingen, waren 1953 Judet et al. [3] die ersten, die zur nicht gelenkübergreifenden Retention eines Fersenbeinbruches ein Drittelrohrplättchen nutzten, was erst später zu Beginn der CT-Ära 1978 von Lanzetta und Meani [4] sowie 1979 von Bèzes und Morilleau [1] wieder aufgegriffen wurde.

Die biomechanische Unzulänglichkeit des Drittelrohrplättchens zur Versorgung von Fersenbeinbrüchen mit Trümmerzonen des Processus anterior calcanei wurde allein dadurch erkennbar, dass zum Beispiel Bèzes et al. [2] in 56 von 120 Fällen das Cuboidgelenk mit dem Drittelrohrplättchen überbrücken und in mehr als einem Drittel ihrer Fälle (45 von 120) eine Y-förmige Doppelverplattung durchführen mussten. Nach vermehrtem, behelfsmäßigem Einsatz von Einfach-, Doppel- und Dreifach-H-Plättchen in der Fersenbeinchirurgie, die eigentlich für die ventrale Spondylodese konzipiert waren, zeigte sich, dass diese zur Abstützung subthalamischer Trümmer- und Impaktionszonen miteinander T- oder gedoppelt H-förmig zusätzlich kombiniert werden mussten $[9,10]$. Um diese Improvisation zu vermeiden, stand erstmals 1991/92 nach den Vorschlägen von Roy Sanders (Tampa, Florida) ein anatomisch angepasstes Calcaneusplättchen (Synthes ${ }^{\circledR}$, USA) im Rahmen einer Pilottestung zur Verfügung. Diese Kleinfragmentplatte oder sog. Sanders-Platte stand seit 1994 in 60 und $70 \mathrm{~mm}$ Länge in Stahl- und Titanversion allgemein zur Verfügung. Dieses eigens für den Fersenbeinbruch geschaffene Plättchen bestach in der Initialphase der Anwendung nicht nur durch Minimierung des Peronealsehnenimpingements (1,2 mm Plättchenstärke), seiner möglichen Titanversion (Option der postoperativen CT-Kontrolle), sondern vor allem durch seine anatomische Anpassung mit dadurch erleichterter Fi- xationstechnik. Mit zunehmender Erfahrung in der operativen Versorgung der Calcaneusfraktur zeigten sich trotz der Vorteile dieses Plättchens die noch ungelösten Fixationsprobleme bei ausgedehnter Zerstörung aller Hauptfragmente, bei großen subthalamischen Impaktionszonen und bereits präoperativ bestehender Osteoporose. Dies zeigte sich vor allem dadurch, dass oftmals die verwendeten 3,5er-Kortikalisschrauben nur in der unmittelbar subthalamischen Zone zum Sustentaculum hin, ganz dorsal im Tuber calcanei oder ganz nah zum Calcaneo-Cuboid-Gelenk hin gelegenen Zonen vermehrter Spongiosadichte ein Halt im Knochen gefunden werden konnte [10]. Zur Optimierung der operativen Calcaneusfrakturversorgung wurde daher unter der Federführung des Erstautors in der Internationalen Foot \& Ankle Expert Group (FAEG) der AO/ASIF-International in Zusammenarbeit mit SUSA (Synthes ${ }^{\circledR}$ USA) ein winkelstabiles Implantat entwickelt. Dieses wurde weltweit erstmals am 4.1.2000 klinisch angewandt (Fallbeispiel 1) und ist in der Stahlversion seit 2002 allgemein erhältlich. Seit November 2003 wird es auch in Titan mit 15\% Molybdän gefertigt (Fa. Mathys) und derzeit klinisch erprobt.

\section{Krankengut}

In der Klinik für Unfall- und Wiederherstellungschirurgie am Universitätsklinikum „Carl Gustav Carus“ der Technischen Universität Dresden wurden in der Zeit vom 1.10.1993 bis zum 31.8.2003 496 Patienten mit insgesamt 553 Frakturen behandelt, davon $89,6 \%$ operativ und $10,4 \%$ konservativ. Das durchschnittliche Alter der Patienten betrug 41,7 Jahre. Das Geschlecht war in 78\% der Fälle männlich, in 22\% weiblich. Bei 57 von 496 Patienten $(11,5 \%)$ bestand ein beidseitiger Fersenbeinbruch. Offene Frakturen befanden sich in 10,2\%, Frakturen mit Kompartmentsyndrom (g 3 ) in 5,5\%, eine Polytraumasituation in 22,2\%. Die CT-Analyse ergab in 87,2 \% intraartikuläre Fraktu- 

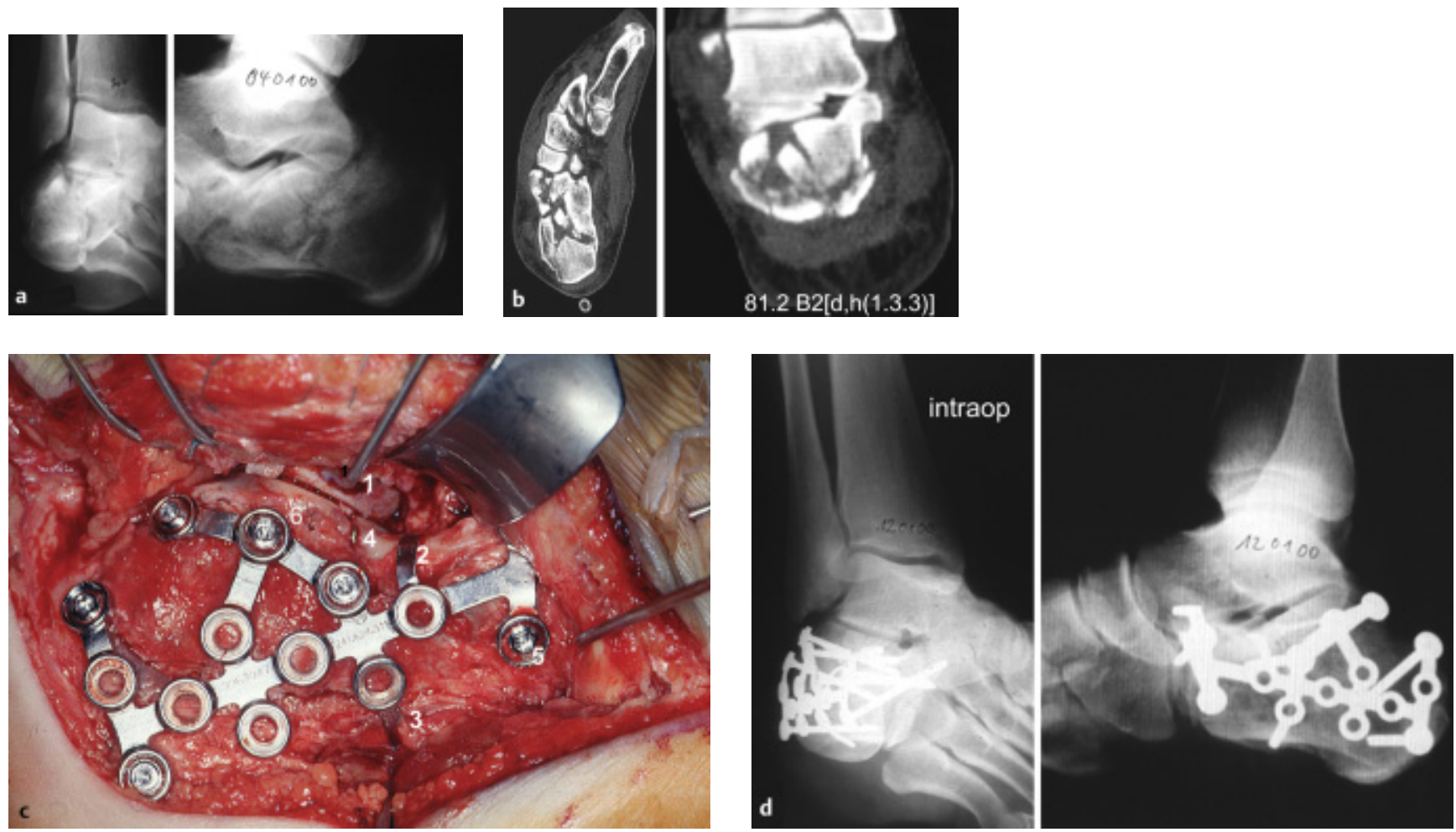

Abb.1 B2-Fraktur des Calcaneus mit zweitgradig geschlossenem Weichteilschaden bei einem 53jährigen Patienten nach suizidalem Fenstersturz. Nach der neuen AO-Klassifikation: 81.2 B2 (d, h [1.3.3]). (a) Die seitliche Calcaneus- und Brodén-30 -Projektion zeigen die tiefe Impaktion der posterioren Facette. (b) Die axiale (links) und koronare CTSchicht (rechts) zeigen die schwere Zerstörung des Calcaneo-CuboidGelenkes sowie des Subtalar-Gelenkes. 81 = Rückfuß, 81.2 = Calcaneus, Icaneus, B2 $=2$-Gelenkfraktur, $\mathrm{d}=$ posteriore Facette, $\mathrm{h}=$ cuboidale Gelenkfläche, 1.3.3 = knöcherne Läsion, multifragmentär, erheblich disloziert. (c) Intraoperativer Situs nach anatomischer Rekonstruktion und Fixation mit der neuen winkelstabilen Calcaneusplatte. $1=$ einer der 2,0er-Spickdrähte im Talus gelenknah umgebogen zum Weghalten

des Weichteillappens, $2=$ kleiner Armausleger zum Herunterhalten des Processus anterior-Fragmentes, $3=$ kleiner Armausleger zum Hochpressen eines möglichen plantarseitigen triangulären Fragmentes, $4=2,0$-Minischraube zur interfragmentären Verschraubung der in sich gebrochenen lateralseitigen posterioren Facette, $5=$ eine der winkelstabilen Schrauben, hier nahe zum Calcaneo-Cuboid-Gelenk, $6=3$,5er-Schraube subthalamisch, die als Zug-Schraube gebohrt durch interfragmentäre Zugwirkung zum Sustentaculum hin eine Spaltbildung vermeiden lässt. (d) Die intraoperative seitliche Calcaneus- und $20^{\circ}$-Brodénaufnahme zeigen die anatomische Rekonstruktion des Subtalar- und Calcaneo-Cuboid-Gelenkes.

ren, die sich in $72,8 \%$ als 4 -Fragment- $/ 1$ Gelenk- bis 5-Fragment-/3-Gelenk-Frakturen erwiesen. Nach dem früher beschriebenen 12-Punkte-Score [9] fanden sich in $58,7 \%$ der Fälle 7-12-PunkteFrakturen.

Die operative Versorgung erfolgte im Mittel 8,8 Tage nach Trauma $(0-23 \mathrm{~d})$. In 95,3\% wurde ein ausgedehnt lateraler Zugang durchgeführt, in $1,5 \%$ eine bilaterale Inzision, in 1,0\% ein ausschließlich medialer Zugang. Eine perkutane, in der Regel arthroskopisch und BV-gestützte Schraubenosteosynthese erfolgte in $2,2 \%$ der Fälle, eine primäre Arthrodese in nur 0,4\%. Die 453 Osteosynthesen wurden in 350 Fällen (77,3\%) mittels Sanders-Titanplättchen durchgeführt, in 28 Fällen (6,2\%) mittels H-Plättchen, als alleinige Schraubenosteosynthese in 22 Fällen (4,8\%). Das winkelstabile Calcaneusplättchen in Stahlversion wurde in 53 Fällen
(11,7\%) verwandt, wobei eine additive Spongiosaplastik in diesen Fällen nur zweimal (3,8\%) notwendig wurde. Bei Verwendung des Sanders-Titanplättchens war hingegen in 53,0\% eine zusätzliche autologe Spongiosaplastik durchgeführt worden.

In den 453 operierten Fällen fanden sich an Komplikationen: Wundrandnekrosen (6,0\%), revisionsbedürftiges Hämatom $(4,7 \%)$, Weichteilinfektion (4,3\%), Knocheninfektion $(2,2 \%)$ und eine verzögerte Knochenheilung in 0,4\% der Fälle.

\section{Winkelstabiles Implantat, warum?}

Wenngleich im 5-Jahres-Follow-up von 194 kontrollierten Fällen nach dem eigenen \pm 200 -Punkte-Score $72 \%$ sehr gute und gute, $25 \%$ befriedigende und 3\% schlechte Ergebnisse erzielt wurden und eine sekundäre subtalare Arthrode- serate von nur 5,6\% feststellbar war, zeigten sich dennoch einige implantatbezogene Probleme, die es zu lösen galt. Bei Verwendung des Sanders-Titanplättchens kam es in 1,1\% der Fälle zum Implantatversagen im Sinne von Schrauben- oder Plättchenbruch, wobei der Einsatz von Endobone in einem Fall, ein Frühinfekt mit Osteonekrose im zweiten Fall und eine unzureichende biomechanische Osteosynthese im dritten von insgesamt 4 Fällen dafür verantwortlich $\mathrm{zu}$ machen waren. Als Nachteil für das nicht winkelstabile Sanders-Plättchen zeigte sich jedoch die sehr hohe Rate von bis zu 53\% an autologer Spongiosaplastik mit einer Vollbelastbarkeit erst ab der 13. Woche. Vereinzelte Fälle einer nicht als ausreichend stabilen Plättchenosteosynthese mit der Sanders-Platte fand sich bei primär gegebener Osteoporose, bei ausgedehnter Trümmerzone in der Calcaneushalsregion und in der cuboida- 

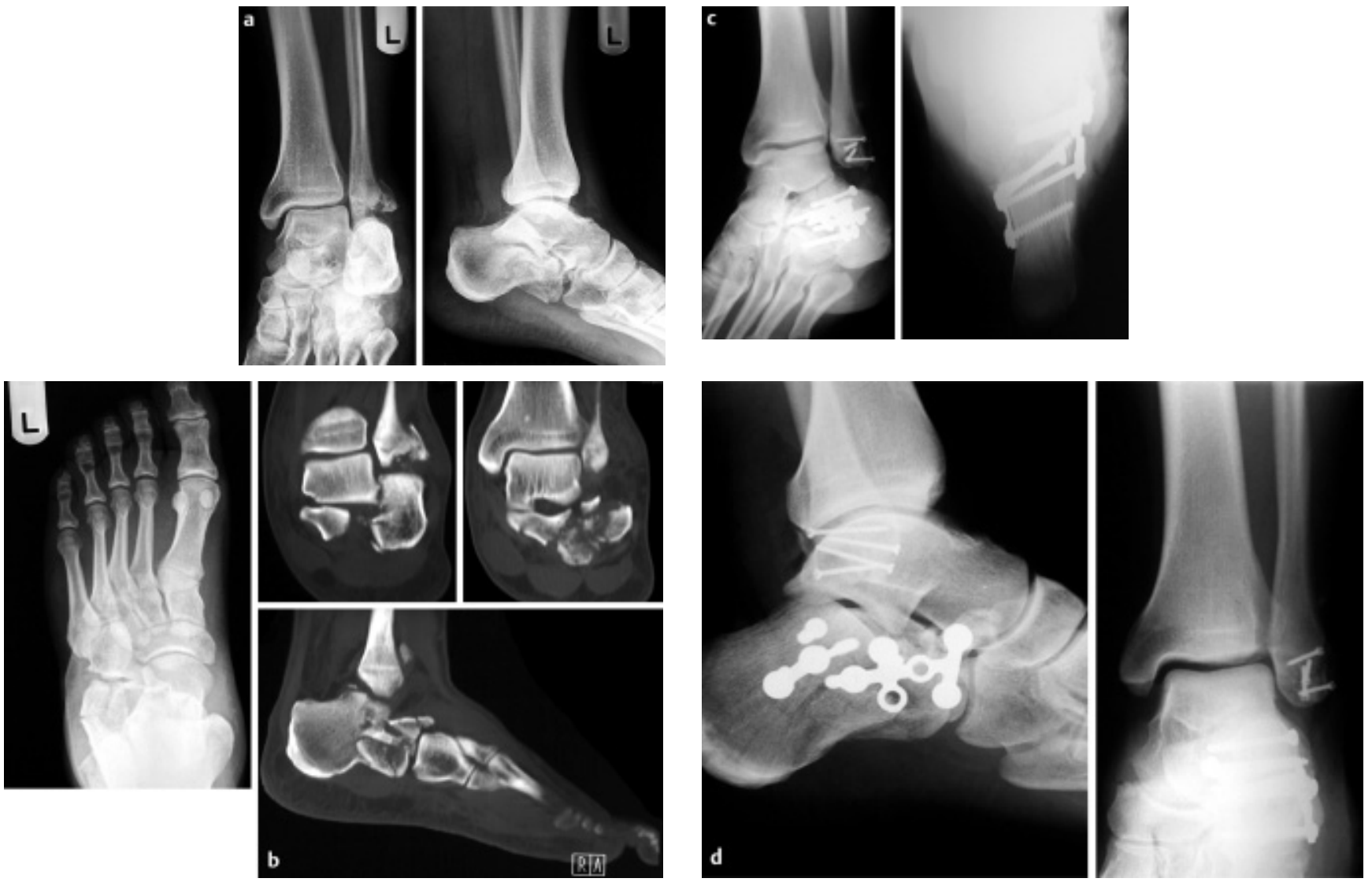

Abb. 2 Erheblich dislozierte C2-Luxationsfraktur des Calcaneus mit zweitgradig geschlossenem Weichteilschaden bei einem 28-jährigen Bierbrauer nach Treppensturz. (a) Die a. p.-Aufnahme zeigt den kompletten Versatz des Fersenbeines nach lateral unter den Außenknöchel mit Zertrümmerung desselben. Die seitliche Projektion zeigt die nach kranial verrenkte Stellung des Calcaneus in Bezug zum Talus und die schwere intraartikuläre Destruktion vor allem zum Cuboid hin. (b) Die dorso-plantare Röntgenaufnahme sowie die weiterführenden CT-Schichten zeigen die schwere Zertrümmerung des gesamten Processus anterior mit erheblicher Verwerfung des Gelenkes. Versatz des Tubers mit der posterioren Facette um über halbe Talusbreite

nach lateral bis unter den Außenknöchel mit Zerstörung desselben in seinem distalen Anteil. Zusätzlicher Frakturverlauf bis nahe zur medialen Gelenkfacette hin. (c) Anatomische Rekonstruktion des SubtalarGelenkes in der intraoperativen Brodén-Projektion mit Rekonstruktion des Außenknöchels mittels Minischrauben. Die axiale Aufnahme zeigt das 2-Loch-winkelstabile-Plättchen auf der Medialseite im Sinne des Antigleitprinzipes. (d) Die seitliche Fersenbeinaufnahme und die OSG a.p.Aufnahme zeigen den lateral platzierten 6-Loch-Anteil der winkelstabilen Calcaneusplatte mit anatomischer Rekonstruktion des Subtalar- und Calcaneo-Cuboid-Gelenkes sowie des Außenknöchelbereiches.

len Gelenkebene des Processus anteriorbzw. anterioren Facetten-Fragmentes. Bei zusätzlicher Frakturierung des sustentacularen Hauptfragmentes in sich und bei ausgedehnten B3-Frakturen des Fersenbeines musste in Einzelfällen $(<2 \%)$ zur Vermeidung eines Repositions- und Retentionsverlustes ein zusätzlicher Gipsverband für 6 Wochen angelegt werden.

Die Notwendigkeit zur Entwicklung eines winkelstabilen Calcaneusplättchens bestand in erster Linie darin, möglichst eine additive Spongiosaplastik zu vermeiden sowie eine primäre Stabilität auch bei zunehmend beobachtbaren Fällen einer primären Osteoporose, bei ausgedehnten B3-Gelenk- und Luxationsfrakturen zu haben.
Die Forderungen an ein winkelstabiles Fersenbeinplättchen bestanden darin, ein Implantat $\mathrm{zu}$ entwickeln, welches möglichst wenig Knochenbedeckung verursacht, d.h. im Wesentlichen, anatomisch angepasst, aus Schraubenlöchern mit Innengewinde besteht, die mit schmalen Armen verbunden sind. Dies deshalb, um möglichst wenig Implantatmasse mit nur geringstem Knochenkontakt und gleichzeitig hoher primärer Stabilität durch die winkelstabile Verschraubung zu haben. Zusätzlich sollten neben den „interlocking screws“ noch gewöhnliche 3,5er- und 2,7er-Schrauben mit dem Plättchen kombinierbar sein, um in bestimmten Bereichen Lage und Richtung der Schraube vom Gewinde unabhängig platzieren zu können. Um das Sustentaculum ohne Zielgerät zu treffen, sollte das Gewinde subthalamisch um 5 Grad nach kranial und 5 Grad nach cuboidal geneigt sein. Ein weiteres Ziel der Entwicklung bestand darin, das Plättchen in situ nachbiegen zu können, was durch kleine Bolzen mit Gewinde ermöglicht werden sollte. Da am Calcaneushals durch den Zug des Ligamentum interosseum eine oftmals hartnäckige Dislokationsneigung nach fußrückenwärts besteht, sollte neben der winkelstabilen Verschraubungsmöglichkeit das Plättchen durch einen zusätzlichen kleinen Arm ergänzt werden, der im Sinus tarsi das Calcaneus anterior-Fragment nach plantar reponiert halten kann (Abb.1). Ein dem Calcaneo-Cuboid-Gelenk naher Processus anterior-Abriss mit ebenfalls beobachtbarer großer Dislokationstendenz nach kranial, dies aufgrund des 

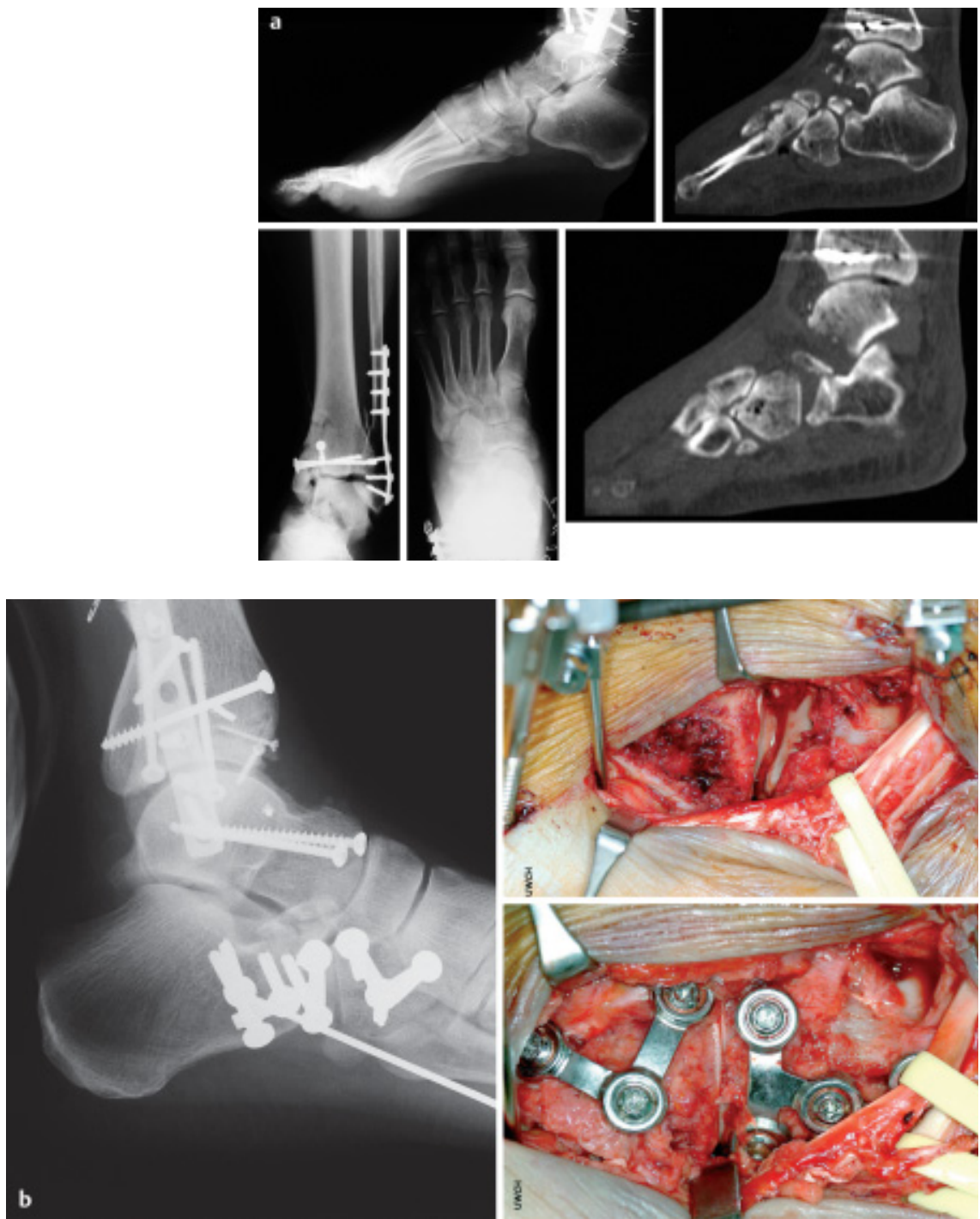

Zuges durch das Ligamentum bifurcatum, sollte noch ein drittes Schraubenloch für diese Region zur Verfügung stehen, welches nach Umbiegen den kranialen Anteil des Processus anterior-Fragmentes nach kaudal pressen kann. Alle diese Forderungen konnten einschließlich sehr glatt schneidender Zangen in einem 7-jährigen Prozess technisch erreicht werden.

Zur Verbesserung der biomechanischen Stabilität des Plättchens einerseits und der postoperativen Möglichkeit einer reflektionsfreien CT-Untersuchung wurde von Anfang an eine Titanlegierung mit Molybdän (15\%) angestrebt und ist seit 11/2003 realisiert. Die Versatilität des Plättchens ist zwischenzeitlich zur exakten anatomischen Anpassung in 3 Größen $(75,7 / 68,8 / 64,5 \mathrm{~mm})$ für rechts und links erhältlich.
Abb.3 Komplexes Fußtrauma mit auswärtig inkongruent versorgter Pilon tibiale-Fraktur mit noch unversorgten Frakturen des Talus, Calcaneus und Cuboids. (a) Erfolgte Korrektur des Pilons in den CT-Schnitten (rechts) und noch unversorgte Talushals- sowie schwere Impaktionsfraktur des distalen Calcaneus mit tiefer Impression der cuboidalen Gelenkfläche in den Calcaneus, zusätzlicher Abriss des eigentlichen Processus anterior calcanei. Mäßige Impaktion der intraartikulären Fraktur des Cuboids. (b) Intraoperativer Situs mit deutlicher Impaktion der cuboidalen Gelenkfläche unter Exposition durch Einsetzen eines Minidistraktors (rechts oben). Die Peronealsehnen sind mit einem Gummizügel weggehalten. Wiederherstellung der calcaneo-cuboidalen Gelenkkongruenz mit Versorgung mittels winkelstabilen Anteilen der interlokking calcaneal plate (rechts unten). Temporäre Transfixation des Calcaneo-Cuboid-Gelenkes mittels K-Draht. Verschraubung des Talus und anatomische, sekundäre Rekonstruktion der Pilonfraktur mit Mini- und 3.5er Schrauben (links).

den in sich extrem geborstenen Processus anterior-Gelenkabschnitt lateralseitig und durch ein zusätzlich abgetrenntes 2-Loch-Segment eine maximale Stabilität im Sinne des Antigleitplättchens medial gewährleistet werden kann.

Das dritte Fallbeispiel zeigt, dass bei komplexem Fußtrauma wie unter anderem bei distaler intraartikulärer CalcaneusLuxationsfraktur kombiniert mit Cuboidfraktur mittels 5-Loch-Plättchen nicht nur der Processus anterior calcanei-Anteil, sondern mit einem abgetrennten 3Loch-Verbund das Cuboid winkelstabil versorgt werden kann. Der deutlich erkennbare Vorteil anatomisch angepasster und winkelstabiler Implantate soll in $\mathrm{Zu}-$ kunft auch für kleinere Fußwurzelknochen wie Talus, Naviculare, Cuboid und Cuneiformia genutzt werden. 


\section{Diskussion}

Wenngleich mit dem winkelstabilen Calcaneusplättchen in 53 Fällen noch kein direkter Vergleich mit der großen Zahl von 350 verwendeten Sanders-Plättchen durchgeführt werden kann, fällt zumindest der drastische Rückgang von additiver autologer Spongiosaplastik von 53 auf $3,8 \%$ auf. Hinsichtlich der bekannten Spendermorbidität erscheint bereits hierin ein Vorteil gegeben. Als geringer Nachteil imponiert das stärkere Auftragen des winkelstabilen Plättchens durch die erhabenen Schraubenlöcher mit Innengewinde von $1,5 \mathrm{~mm}$ gegenüber den $1,2 \mathrm{~mm}$ flachen Sanders-Plättchen. Mit dem von der Fa. Litos angebotenen Calcaneusplättchen aus Reintitan mit der später entwickelten Option winkelstabil einzudrehender (2 differierende Härtegrade) Schrauben, liegen keine eigenen Erfahrungen vor. Auch finden sich in der Literatur keine Angaben, ob diese Implantate problemlos zu entfernen gehen. 12 von 53 winkelstabilen Fersenbeinplättchen (Stahlversion) des eigenen Krankengutes konnten problemlos entfernt werden. Eigene experimentelle Untersuchungen zum direkten Vergleich der primären Stabilität bei Verwendung von winkelstabilen Plättchen vs. SandersPlatte an instabilen B1-Cadaver-Calcanei stehen noch aus.

\section{Literatur}

${ }^{1}$ Bèzes H, Morilleau Ph. Ostèosynthèse des fractures thalamiques du calcanéum avec enfoncement thalamique par plaque visée „1/3 tube“ (d'après 75 observations). 4èmes Journées Militaires de Chirurgie Orthopédique, Hôpital Laveran, Marseille, 1979; p 75

${ }^{2}$ Bèzes H, Massart P, Fourquet JP. Die Osteosynthese der Calcaneus-Impressionsfraktur. Unfallheilkunde 1984; 87: 363-368

${ }^{3}$ Judet R, Judet J, Lagrange J. Traitement des fractures du calcanéum compartant une disjunction astragalocalcanéenne. Mem Acad Chir 1954; 80: 158 - 160

${ }^{4}$ Lanzetta A, (1975) Meani E. Utilisation des plaques pour fracture du calcanéum. Nouv Presses Méd 1977; 33: 2985-2986

${ }^{5}$ Lenormant C, Wilmoth P. A propos du traitement sanglant des fractures du calcanéum. Bull. Mém. Soc. nat. Chir. 1928; 54: 1354

${ }^{6}$ Leriche R. Traitement chirurgical des fractures du calcanéum. Bull Mem Soc Nat Chir 1929; 55: 8-9

${ }^{7}$ Merle d'Aubigné MR. Fracture isolée de la petite apophyse du calcanéum traitée par ostéosynthèse. Mém Acad Chir (Paris) 1936; 62: 1155-1159

${ }^{8}$ Westhues H. Eine neue Behandlungsmethode der Calcaneusfrakturen. Zentralbl Chir 1935; 62: 995-1002

${ }^{9}$ Zwipp H, Tscherne H, Wülker N, Grote R. Der intraartikuläre Fersenbeinbruch: Klassifikation, Bewertung und Operationstaktik. Unfallchirurg 1989; 92: 117-129

${ }^{10}$ Zwipp H. Der intraartikuläre subthalamische Fersenbeinbruch. Operat Orthop Traumatol 1995; 7 (Heft 4): 237-249

Prof. Dr. med. Hans Zwipp

Ärztlicher Direktor

Dr. med. Stefan Rammelt

Funktionsoberarzt

Dr. med. Sven Barthel

Assistenzarzt

Klinik und Poliklinik für Unfall- und Wiederherstellungschirurgie am Universitätsklinikum „Carl Gustav Carus“ der Technischen Universität Dresden

Fetscherstraße 74

D-01307 Dresden 